MODELOS ESTÉTICOS EN LAS CIENCIAS HUMANAS

Un estudio epistemológico

Lorenzo Bartalesi 


\section{Lorenzo Bartalesi}

Scuola Normale Superiore di Pisa

Modelos estéticos en las ciencias humanas: un estudio epistemológico

DOI: $10.36446 /$ be.2020.51.142

\section{Resumen}

Lo estético es un hecho antropológico que - como el lenguaje o el pensamiento simbólico- pertenece al registro comportamental, cognitivo y social de nuestra especie. A partir de esta consideración, el artículo pretende aclarar los usos de la categoría de lo "estético" en las ciencias humanas (antropología social, psicología cognitiva, antropología evolutiva). La investigación epistemológica se concentra en los supuestos implícitos que guían las diferentes metodologías y enfoques teóricos, y finalmente propone un mapa conceptual de los modelos estéticos adoptados en el debate contemporáneo. Este resultado se presenta como un paso analítico preliminar destinado a promover un enfoque interdisciplinario de la complejidad y diversidad sociobiológica de los fenómenos estéticos.

\section{Palabras clave}

Estética; Antropología de lo estético; Ciencias humanas; Paradigmas epistémicos

\section{Aesthetic models in the human sciences: an epistemological study}

\section{Abstract}

Starting from the assumption that aesthetic is an anthropological fact which - like language or symbolic thought - belongs to the behavioral, cognitive and social register of our species, the article aims to clarify the uses of the category of aesthetic in the human sciences (social anthropology, cognitive psychology, evolutionary anthropology). The epistemological analysis focuses on the implicit assumptions that guide the different methodologies and leads to the elaboration of a conceptual map of the several models of aesthetic adopted in the contemporary debate. Such a result is presented as a preliminary analytical step aimed at fostering an interdisciplinary approach to the complexity and socio-biological diversity of aesthetic phenomena.

\section{Keywords}

Aesthetics; Anthropology of Aesthetics; Human Sciences; Epistemic Paradigms

Traducido del italiano por Facundo Bey (CONICET)

Recibido: 27/03/20. Aprobado: 24/04/20.
El artículo se propone clarificar los usos de la categoría de estética en las ciencias humanas contemporáneas. ${ }^{1}$ Con la expresión "ciencias humanas" entendemos aquí el conjunto de disciplinas que comparten la ambición de elaborar "un sistema de interpretación que explique simultáneamente los aspectos físicos, fisiológicos, psíquicos y sociológicos de todas las conductas" (Lévi-Strauss 1950: 25). Cada vez es más evidente que la definición clásica de las ciencias humanas como Geisteswissenschaften plantea problemas teóricos insuperables. Los recientes avances en el conocimiento de la plasticidad cerebral o de los procesos epigenéticos, así como una revisión crítica del paradigma naturalista de la modernidad occidental (Descola 2005), delinean un horizonte teórico nuevo en el que la naturaleza y la cultura son polaridades fluidas, expuestas a la posibilidad de mutar la una en la otra en los tiempos profundos de la evolución, sugiriendo así una sustancial superación de la tradicional oposición entre nature y culture.

La arqueología, la antropología social y las demás ciencias humanas han reconocido tradicionalmente el papel de los fenómenos estéticos en las culturas humanas y, a pesar de la naturaleza indeterminada de la estética filosófica occidental, ${ }^{2}$ han recurrido ampliamente

\footnotetext{
1 Este artículo propone una síntesis (y espero un pequeño avance) de una investigación epistemológica sobre lo estético en cuanto hecho antropológico que encontró una primera formulación en Bartalesi 2017.

2 Como recuerda Emilio Garroni, la estética siempre ha oscilado entre "la existencia y la no existencia de un objeto epistémico propio, entre la teoría del arte y la comprensión a través del arte, entre la estética como ciencia o filosofía del arte y la estética como filosofía crítica" (Garroni 1992: 38).
} 
a las categorías elaboradas por esta última para investigar fenómenos expresivos complejos como la figuración o la acción ritual. Sin embargo, esta adopción no carece de consecuencias teóricas y está a la base de malentendidos conceptuales y de problemas epistemológicos.

\section{EL IMPLÍCITO MODERNISTA}

Desde los albores de la antropología social, la categoría de estética ha sido un componente esencial del estudio de las culturas humanas. Ejemplar es el largo capítulo dedicado a la estética por Marcel Mauss en su Manuel d'ethnographie (Mauss [1926] (1967): 85) o el recurso de Franz Boas a la noción de "valores estéticos" en su estudio del arte primitivo (Boas [1927] (1951): 9). Aún más evidente es el papel de la estética en el desarrollo de la arqueología como disciplina autónoma. Sobre todo en el ámbito de los estudios sobre el arte clásico griego y romano de inspiración winckelmanniana, no sólo la apreciación estética ha sido central durante mucho tiempo en la asignación de un valor a los artefactos, sino también las mismas categorías de la estética han representado un componente importante del bagaje teórico disciplinar.

Sin embargo, en los últimos treinta años, en ambas disciplinas, la categoría de estética fue sometida a una crítica radical que ha conducido progresivamente a su marginación y, en algunos casos, a su abandono. ${ }^{3}$ En el origen de este proceso se encuentra el reconocimiento a la estética de un compromiso normativo no integrable dentro de los procesos de validación de la disciplina reformada sobre una base científica. La estética correspondería al espacio pres-

${ }^{3}$ Véase, por ejemplo, la remoción de la noción de arte en la arqueología: Conkey y Hastorf 1990, White 1992. criptivo de los juicios de valor etnocéntricos y, por tanto, reconocer su carácter universal tendría el efecto de producir un profundo malentendido de las conductas y de las categorizaciones no occidentales. Como afirma lapidariamente la antropóloga Christina Toren, "la estética no es una categoría analítica, ni jamás será tal. Es completamente innecesario. No lo necesitamos para estudiar las máscaras de Abelam, o las pinturas de Yolngu, ni cualquier otra cosa" (Ingold 1996: 230).

Si, por una parte, la dimensión constitutivamente valorativa de los hechos estéticos es innegable, por otra parte, es del todo errónea una estrategia que lleve a tirar al niño junto con el agua sucia de su baño. La asunción presente en la base de la remoción de la estética se debe principalmente a una confusión entre el fenómeno investigado y la entidad teórica que lo tematiza o, aún más radicalmente, a la identificación impropia del dominio estético con una "teoría especulativa del arte” (Schaeffer 1992) fundada en el ideal romántico de un arte sacralizado, desinteresado y autónomo que, durante casi dos siglos, ha legitimado ideales estéticos como el esteticismo y el art pour l'art. Esta doctrina "modernista" representa la realización del mismo proceso histórico que, a partir del siglo XVIII, ha hecho del arte una provincia de la estética (Talon-Hugon 2014) y que ha reducido al mismo tiempo la multiforme variedad antropológica de los fenómenos estéticos a un estado psicológico contemplativo, idiosincrático y centrado sobre las meras propiedades formales de una obra de arte. En palabras de Pierre Bourdieu, atento analista y crítico radical de esta doctrina, es una forma de estética pura que "tiene sus raíces en una ética o, mejor aún, en un ethos, de distancia electiva con respecto a las necesidades del mundo natural y social, que puede tomar la forma [...] de un esteticismo, el cual, al constituir la disposición estética en principio de aplicación universal lleva la negación burguesa del mundo social a su límite" (Bour- 
dieu 1979: VI). Más que exaltar la complejidad variada de los fenómenos estéticos, esta doctrina revela una concepción residual de lo estético como componente accesorio de las culturas. Ornamental, seductora, asemántica, afectiva, idiosincrática, el carácter penetrante del ideal estético modernista se refleja hoy en la anestetización del hombre, propia de las sociedades postindustriales, y en una fragmentación del debate sobre la estética en el seno de las ciencias humanas contemporáneas.

\section{MALENTENDIDOS Y PARADOJAS}

La doctrina estética modernista es adoptada por la mayoría de las ciencias humanas contemporáneas algunas veces como objetivo polémico (Elkins y Montgomery 2013), pero, más a menudo, como asunción implícita no verificada. Un caso paradigmático es el intenso debate en el seno de la antropología social entre quienes, como hemos visto, le niegan legitimidad teórica a la categoría de estética y quienes, desde una posición minoritaria, defienden su carácter transcultural. Este es el caso de Howard Morphy según el cual "la estética está relacionada con todo el proceso de socialización de los sentidos con la evaluación de las propiedades de las cosas. [...] La capacidad humana de transformar las propiedades físicas en valoraciones estéticas es esencial para comprender la acción y la elección humana en contextos contemporáneos y evolutivos" (Morphy 1996: 209). El análisis de diversas posiciones en el debate nos confirma que la actual remoción de la estética se debe precisamente al arraigo de la doctrina modernista en el bagaje teórico de la antropología social (en particular, en la tradición funcionalista anglosajona). Según Alfred Gell todas las dificultades a las que se ha enfrentado tradicionalmente la antropología del arte surgen de una promoción de la estética desde el discurso filosófico occidental hasta un conjunto de creencias sobre hechos del mundo real. Por lo tanto, para escapar del etnocentrismo la antropología social debe abandonar "la idea de que los objetos están relacionados con los agentes sociales «al modo del arte» si (y solo si) los agentes sociales consideran a estos objetos «estéticamente»" (Gell 1998: 13). Para ello, propone Gell, es necesario adoptar un «filisteísmo metodológico» que "consiste en tomar una actitud de firme indiferencia hacia el valor estético de las obras de arte - el valor estético que tienen, ya sea de forma autóctona o desde el punto de vista del esteticismo universal" (Gell 1992: 42).

El malentendido de Gell -y de toda posición antiestética construida sobre los mismos argumentos- es, pues, negar la factualidad de lo estético en virtud de la incompatibilidad epistémica de la estética con las culturas no occidentales. El malentendido toma las formas de una confusión entre dos tipos de problemas: la cuestión de la «pertinencia» teórica de la categoría de estética para la disciplina y la de la «transculturalidad» de una clase específica de fenómenos humanos. Si la cuestión de la pertinencia de las categorías de la estética filosófica concierne al proceso interno de constitución de un metalenguaje para el discurso antropológico, por el contrario, el estudio de los comportamientos "estéticos" es tarea de una investigación empírica y comparativa. Si en el primer caso el análisis conceptual se enfrenta con los problemas que plantea la adopción de categorías occidentales en un contexto externo, en el segundo, la investigación comparativa tiene por objeto circunscribir la identidad de un fenómeno humano más allá de las fronteras culturales. Si bien es innegable que la utilización de las categorías adoptadas deforma el fenómeno estudiado $y$, en consecuencia, que el metalenguaje de la antropología es un componente central de la investigación comparativa, también es cierto que la remoción del fenómeno investigado empíricamente no se sigue de la falta de pertinencia de una categoría etnocéntrica. Del fracaso o inadecuación de una enti- 
dad teórica no deriva la inexistencia del fenómeno por ella tematizado. ${ }^{4}$ A lo sumo, de ello se sigue una revisión de la categoría con el fin de restituir con la máxima elegancia y simplicidad descriptiva el fenómeno en su complejidad y autenticidad. Si una versión calibrada de filisteísmo metodológico constituye ciertamente un útil principio de neutralidad axiológica, es necesario reafirmar que la extensión del espacio de los fenómenos estéticos humanos no corresponde al espacio epistémico de las entidades teóricas de la modernidad occidental.

La desconfianza demostrada hacia la estética por gran parte de la antropología social y de la arqueología contemporáneas es diametralmente opuesta al interés creciente demostrado por las ciencias humanas "naturalistas" como la psicología, la antropología evolutiva o los estudios neurofisiológicos de la cognición humana. En estos campos de investigación asistimos a una auténtica proliferación de investigaciones experimentales sobre las dinámicas perceptivas en el régimen estético, sobre los fundamentos neurobiológicos de las experiencias estéticas o sobre la historia evolutiva de las preferencias estéticas humanas. Si miramos a los tratamientos reservados por las ciencias humanas a los fenómenos estéticos en los últimos treinta años observamos una situación paradójica: marginada por las ciencias humanas "cualitativas" por la inconciliabilidad de su versión modernista con un enfoque científico con respecto a las culturas humanas, lo estético se ha convertido en objeto epistémico de extremo interés para las ciencias humanas "cuantitativas" precisamente por la compatibilidad del formalismo modernista con las metodologías experimentales.

\footnotetext{
${ }^{4}$ Para un uso eficaz de este tema en apoyo del papel de la estética en la explicación arqueológica, véase Currie (2016): 233-246.
}

Caso ejemplar de esta situación paradójica es el programa estándar de la estética evolutiva, tal como se ha venido delineando en los últimos veinte años. Como se resume en el único manual de estética evolutiva publicado hasta la fecha, el objetivo principal de este programa es "la importación de la estética en las ciencias naturales, y especialmente su integración en la heurística de la teoría evolutiva de Darwin" (Voland y Grammer 2003: 5). En síntesis, se trata de "importar" la teoría estética modernista en el programa de la psicología evolutiva centrado en el patrimonio genético, sobre el concepto de adaptación y sobre un modelo de mente computacional y modular (Barkow, Cosmides, Tooby 1992). El objetivo de esta perspectiva es reconstruir la historia evolutiva de las preferencias estéticas humanas y de las estructuras biológicas que las implementan. Dejando de lado muchos de los problemas epistemológicos relacionados con querer importar una doctrina histórica dentro de una explicación evolutiva, aquello que parece evidente es el radical empobrecimiento de la categoría de lo estético. La complejidad de los comportamientos estéticos resulta identificada con el conjunto de comportamientos de discriminación y selección de rasgos ambientales y/o sexuales. En la reducción de la estética a una teoría de las preferencias (Bartalesi 2013: 12-13), facilitada precisamente por la adopción de una concepción modernista de lo estético, el modelo estándar de estética evolutiva termina por perder de vista la complejidad real de la dimensión estética de la experiencia como red de reacciones instintivas, emociones, sentimientos, pensamientos y juicios, cuyas preferencias constituyen sin duda uno de los componentes, pero no el único ni el fundamental. ${ }^{5}$

\footnotetext{
${ }^{5}$ Para una crítica del programa estándar de la estética evolutiva véase Portera y Bartalesi 2016.
} 


\section{HECHOS ANTROPOLÓGICOS Y MODELOS ESTÉTICOS}

En este punto, es necesario delinear una noción de lo estético que, además de respetar el principio de neutralidad axiológica exigido por Gell, vaya en la dirección de una integración de las diferentes perspectivas disciplinarias y de una disolución de los malentendidos resultado de la adopción de ideales estéticos prescriptivos. El punto de partida no puede sino ser la afirmación según la cual la actitud estética humana es un «hecho antropológico» que - al igual que el lenguaje o el pensamiento simbólico - pertenece al registro comportamental y cognitivo de nuestra especie. Tal como sucede con cada una de nuestras otras características biológicas, los comportamientos estéticos tienen una larga historia evolutiva que se remonta al menos a los primeros milenios del Paleolítico Inferior. ${ }^{6}$ Éstos participan en todos los momentos cruciales de la existencia de un individuo, en diversas formas y modalidades, y están presentes en todas las latitudes y en todos los contextos culturales. Es en el corazón de la evolución humana y de las sociedades donde encontramos en obra a lo estético, no sólo como producción artística u ornamental, sino también como dinámica cognitiva específica en juego en el aprendizaje (Dissanayake 2000), en la creación de artefactos, en las prácticas de socialización cruciales para la transmisión cultural (Hobart y Kapferer 2005).

Inicialmente podemos definir lo estético como una serie de procesos cognitivos que se desencadenan en el momento de la percepción de eventos u objetos con determinadas características. Estos proce-

\footnotetext{
${ }^{6}$ Pero con toda probabilidad se remonta a una era mucho más antigua. El debate es muy vivaz en arqueología. Una voz autorizada en apoyo de un datación retrospectiva de las huellas de lo estético en la evolución humana es la de Francesco d'Errico y su equipo (D'Errico y Henshilwwod, 2011). Para un reconocimiento general, véase Lorblanchet y Bahn 2017.
}

sos, que incluyen la atención, la inversión emotiva y el juicio, producen una organización significativa de las cualidades sensoriales del mundo en una experiencia específica, y son la base de aquellas conductas estéticas humanas complejas a través de las cuales las instituciones, códigos éticos, creencias religiosas, son percibidas, comprendidas y transmitidas. Aunque sea mínima, esta primera definición es suficientemente exhaustiva, ya que recoge casi todas las principales acepciones del término "estética" adoptadas en las ciencias humanas:

i. un funcionamiento específico de los procesos cognitivos, afectivos y somáticos;

ii. una forma de experiencia que se distingue del flujo ordinario de la percepción;

iii. un conjunto de elementos expresivos — cantos, danzas, esquemas formales, artefactos- que desencadenan tal forma de experiencia;

iv. las preferencias estéticas, a través de las cuales los seres humanos formulan juicios, hacen elecciones y se orientan en su entorno;

v. la objetivación de las preferencias estéticas en estilos culturales que luego se convierten en objeto de transmisión;

vi. la integración de las preferencias individuales en valores estéticos compartidos que constituyen una jerarquía cualitativa que se traduce en un juicio estético (crítica de arte, estética indígena, etc).

La "sintaxis de lo estético" o la articulación específica de los elementos que componen un fenómeno estético - experiencias, actos expresivos, preferencias, juicios, estilos culturales, etc.- es extremadamente variada; tanto es así que debemos asumir goodmanianamente estos elementos descriptivos más como los “síntomas de lo 
estético"7 que como caracteres esenciales. Tomados en su conjunto, estos elementos componen una definición de lo estético como hecho antropológico multidimensional arraigado en los procesos cognitivos y comportamentales funcionales a la supervivencia de un individuo y de una sociedad (mecanismos cognitivos, reacciones afectivas, emociones, sentimientos, memoria, aprendizaje, hábitos culturales). Síntesis de disposiciones innatas y líneas de desarrollo cultural así como de contenidos emotivos y discriminativoscategorizantes de la percepción, lo estético se presenta mediante una suspensión de las actividades ordinarias, distinguiéndose de otras conductas humanas por su carácter autotélico, endógeno y energéticamente costoso. En sintonía con las cualidades afectivas de una situación dada y alternativa al conocimiento proposicional e inferencial, lo estético es una articulación primaria aunque ya significativa de la complejidad sensorial de un ambiente. Desde el punto de vista de su funcionamiento cognitivo, la atención estética consiste, pues, en un "estilo cognitivo divergente" (Schaeffer 2015b: 104) que privilegia la complejidad contextual del estímulo y actúa como una intensificación de la percepción ordinaria en la que los objetos o los acontecimientos adquieren una significación emocionalmente saturada y cognitivamente indeterminada. Por su peculiaridad fenomenológica, las experiencias estéticas están indisolublemente ligadas a situaciones en las que está en juego nuestro equilibrio emocional y la armonía con el mundo, así como nuestra relación con la trascendencia, con la muerte y con la necesidad de atribuir un significado a la existencia. Por lo tanto, desde un punto de vista fenomenológico, las experiencias estéticas tienen rasgos en común con los "comportamientos que hacen especiales a las cosas" [ma-

\footnotetext{
7 "Un síntoma no es una condición necesaria ni suficiente, sino que simplemente tiende a estar presente junto con otros síntomas en la experiencia estética" Goodman (1968): 252.
}

king special behaviours] descritos por la antropóloga Ellen Dissanayake (2013).

Esta definición antropológica de lo estético puede ser adoptada con provecho por las múltiples metodologías y perspectivas teóricas de las ciencias humanas. El objetivo es favorecer un enfoque interdisciplinario basado en un modelo «estético compartido» capaz de dar cuenta - desde un punto de vista cognitivo y comportamental, social e individual, histórico y evolutivo- de la complejidad y diversidad sociobiológica de los fenómenos estéticos.

En el sentido más amplio y general aquí adoptado, un modelo es el producto de una modelización o bien una transposición a escala de un hecho real. Una reconstrucción fruto de una actividad de selección de características estables y generales de una serie de fenómenos aislados, difusos y discretos que tiene la tarea de representar un fenómeno dado según una función «homeomorfica», de manera que su funcionamiento pueda dar cuenta de todos los hechos observados. Recuperando en parte la acepción estructuralista, ${ }^{8}$ un modelo es el resultado de una doble operación de «descomposición» analítica del fenómeno complejo en un número finito de elementos y de una «reconfiguración» según determinadas reglas constructivas. Lo que un modelo estético ofrece es entonces una configuración que, al poner de relieve los vínculos y la recursividad operativa entre diversos elementos, circunscribe eficazmente el espacio de lo estético.

\footnotetext{
${ }^{8}$ Veáse Barthes 1964 y el precioso análisis de Schaeffer (2015a: 56-76). Sobre la noción de "modelo" (mecánico o estadístico) en un entorno estructuralista, la referencia obligada es a Lévi-Strauss 1958.
} 
Las diversas posiciones que componen el debate contemporáneo sobre la naturaleza de lo estético hacen uso de un gran número de modelos estéticos. Una de las principales tareas de la presente reflexión será, pues, identificar las peculiaridades y las características comunes a estos modelos y comprender si los elementos compositivos adoptados constituyen una base sólida para el conocimiento del fenómeno modelizado o si más bien representan la perjudicial consecuencia de la asunción de implícitos teóricos que derivan del paradigma teórico adoptado. La situación de disgregación en la que se encuentra la investigación sobre los hechos estéticos, incapaz de encontrar la forma unitaria y coherente de un verdadero programa de investigación interdisciplinar, puede ser vista como el resultado de la adopción inconsciente por parte de las ciencias humanas de implícitos conceptuales como reglas constructivas de modelos estéticos alternativos. El resto de la contribución se dedicará, por tanto, a obtener algunos elementos compositivos de los modelos estéticos. En particular, la atención se centrará en tres de ellos: la «concepción de lo estético», el «paradigma epistémico» y la «versión de la mente».

\section{LA CONCEPCIÓN DE LO ESTÉTICO}

¿Qué es un hecho estético en cuanto objeto epistémico? ¿Los hechos estéticos son hechos perceptivos? ¿o pertenecen al orden discursivo? ¿o son también reacciones emocionales? De las respuestas que damos a preguntas como éstas depende la asunción de una específica concepción de lo estético y la consiguiente naturaleza de la relación que los hechos estéticos mantienen con el conjunto de los demás hechos. ${ }^{9}$

\footnotetext{
${ }^{9}$ Para una investigación epistemológica de la compleja noción de "hecho estético" véase Di Liberti 2017.
}

La historia de la estética filosófica está caracterizada por la oposición entre una concepción «emotivista», que identifica lo estético con el contagio afectivo producido por un objeto o un acontecimiento sobre el sujeto que percibe; y una concepción «cognitivista», que piensa lo estético como una forma peculiar de cognición según el modelo del conocimiento inferencial. El campo de la estética filosófica es, pues, terreno de conflicto entre quienes ven en los hechos estéticos una dimensión idiosincrática de la vivencia subjetiva y quienes, por el contrario, identifican la dimensión estética con una forma específica de conocimiento que, análogamente a la racional, está dotada de reglas de correcta aplicación de predicados a objetos del mundo. Como un dilema del que la filosofía parece estar genéticamente afligida - podríamos situar su origen ya en el siglo IV a.C., en el conflicto que opone Platón a los sofistas-, el dualismo emotivismo-cognitivismo acompaña el nacimiento mismo de la estética como disciplina filosófica. Si el siglo XVIII bautiza la estética, esto ocurre precisamente en la comparación entre el racionalismo metafísico de la estética como ciencia del conocimiento sensible de Alexander Gottlieb Baumgarten, y la tradición del empirismo angloescocés, que encuentra su máxima expresión en David Hume y en los discípulos de Shaftesbury, James Arbuckle y William Melmoth.

Figuras clave del emotivismo estético son sin duda los dos filósofos ingleses Clive Bell (1914) y Alfred J. Ayer (1936). Si al primero debemos la célebre identificación de la experiencia estética con la «emoción estética» suscitada únicamente por los valores plásticos de la obra de arte despojados de toda significación (Significant form), al segundo, por su parte, hay que atribuirle la eliminación de la función cognoscitiva de lo estético. Distinguiendo entre una dimensión «normativa» del juicio -indefinible e inverificable- y una «descriptiva» - aserciones empíricas con un contenido fácti- 
co-, el emotivismo de Ayer llega a la tesis del carácter inverificable de las afirmaciones estéticas, las cuales son comparables a una forma interjectiva como "un grito de dolor o una palabra de mando" (véase Kivy 1992). El emotivismo relega por tanto lo estético a la pura interioridad emotiva, que deviene objeto de una ciencia empírica como la psicología, produciendo la separación de la esfera estética de las dinámicas unitarias de la experiencia y del espacio público de las prácticas sociales. Resulta evidente entonces que el emotivismo de Bell y Ayer constituya un momento fundamental de esa doctrina modernista que hemos visto encajar con otros elementos (y de ahí los resultados diametralmente opuestos) para dar vida al modelo estético de la antropología social y al de la estética evolutiva.

La idea baumgartiana de la estética como acuerdo de las representaciones sensibles según las leyes de la razón tomó su forma en el debate contemporáneo de una teoría cognitivista según la cual la cognición estética opera a través de representaciones internas imágenes mentales, conceptos, proposiciones- siguiendo criterios de correcta aplicación de los predicados estéticos. Una versión «débil» del cognitivismo se limita a pensar la relación estética como un tipo específico de proceso de categorización y adquisición de información contenida en un estímulo sensorial. Una concepción tal de lo estético, unida a la asunción de una relación de dependencia causal entre estímulo externo y representación mental, está a la base de los paradigmas en neuroestética y estética experimental que identifican la percepción estética con el reconocimiento de patrones estéticos universales. De una manera más radical, una versión «fuerte» de cognitivismo estético — surgida en el ámbito analítico en respuesta a las tesis eliminatorias de Ayer y del neopositivismo lógico- toma como modelo la actividad crítica y la forma proposicional del juicio cognoscitivo con el fin de identificar criterios de validez objetiva y estrategias de validación enunciativa para los juicios estéticos (Kieran y Mciver Lopes 2006). La experiencia estética es descrita por lo tanto como una forma de conocimiento sujeto a validación epistémica, una elección consciente de opciones lingüísticas o interpretativas ${ }^{10}$ justificadas por la calidad de objetos y acontecimientos del mundo que tiene el valor de un conocimiento testimoniable (Robson 2012), adquirible sin experiencia personal.

\section{EL PARADIGMA EPISTÉMICO}

Los modelos estéticos adoptados por las ciencias humanas no se distribuyen sólo sobre el eje que opone las dos concepciones de lo estético. Ignorando el horizonte teórico transdisciplinar que se viene afirmando cada vez más, las perspectivas antropológicas sobre lo estético continúan disponiéndose sobre las dos placas de una falla epistémica que, por un lado, ve el espacio de los hechos físicos regido por leyes causales y, por otro lado, el dominio de los hechos culturales. Una dicotomía radical que opone los programas de naturalización de lo estético a las perspectivas culturalistas o hermenéuticas. Ambas perspectivas coinciden en el carácter transcultural de lo estético, pero se oponen con respecto al significado que debe darse a la noción de «hecho antropológico».

Para la perspectiva culturalista, lo estético es una «constante» cultural. Esta asunción no implica ninguna afirmación de tipo innatista y es el resultado de una investigación «comparativa» que, si bien establece el carácter transcultural de lo estético, pone el acento sobre la diversidad de las manifestaciones locales. Al contrario, para la

\footnotetext{
10 Un caso ejemplar es la estética ambiental de Allen Carlson, en la cual el conocimiento de conceptos científicos es una condición necesaria para justificar un juicio estético sobre un fenómeno natural (Carlson 1999).
} 
perspectiva naturalista, lo estético es un «universal» biológico. Tal asunción presupone que lo que es definido como antropológico dependa de una serie de disposiciones innatas y universales de nuestra especie, adquiribles mediante investigaciones experimentales.

Para los partidarios de un paradigma culturalista, lo estético es un producto cultural que puede depender para su realización de su sustrato físico pero que es independiente por su variabilidad y calidad emergente. En su forma más radical esta tesis plantea una discontinuidad radical entre el funcionamiento de la cognición estética y su diferenciación en conductas y preferencias culturalmente determinadas. Precisamente en virtud de una supuesta prioridad constructiva de la cultura sobre los componentes innatos (genéticos, cognitivos y comportamentales), esta posición considera que los datos experimentales no son pertinentes para una comprensión de los fenómenos estéticos.

La disciplina donde está mayormente presente una orientación culturalista es naturalmente la antropología social en la que podemos delinear tres principales enfoques teóricos con respecto a lo estético. El primero es un enfoque "prescriptivista» en el que la estética se identifica con el uso de conceptos estéticos y con la presencia de una teoría estética explícita. La investigación, predominantemente de tipo etnográfico, se centra en el hallazgo de "estéticas locales" mediante el estudio de los juicios estéticos en las culturas no occidentales (Thompson 1971; Lawal 1974; Vogel 1980). El segundo es un enfoque "histórico-artístico» en el que el dominio de la estética se superpone al de la producción artística. El resultado es una antropología del arte acuñada sobre los métodos y objetivos de la historia del arte occidental (Anderson 1990). Las investigaciones se centran en un análisis estilístico de los artefactos en busca de una "ley estética" universal o de "principios estéticos" (ritmo, croma- tismo, estructuras formales) específicos de una cultura. Por último, un enfoque «formalista» que, inspirado en la idea boasiana de la estética como "sentimiento por la forma", centra la atención en la calidad de una obra de arte en cuanto capaz de activar una experiencia afectivamente connotada (Maquet 1986). El marco radicalmente formalista de estas investigaciones asume implícitamente la oposición entre la percepción estética, dirigida a una presencia puramente aspectual del objeto y la percepción ordinaria, la cual implica siempre una referencia al espacio público de los significados.

En el frente opuesto de los partidarios de un paradigma naturalista, lo estético es una constante antropológica "porque» lo estético es un universal biológico. En su formulación radical este paradigma se presenta como un programa de naturalización comprometido en una reducción del nivel epifenoménico de las constantes culturales a los sistemas subpersonales de los cuales dependen causalmente (relaciones neuronales, módulos cognitivos, secuencias motoras, adaptaciones). Una perspectiva naturalizante asume axiomáticamente la unidad psíquica y cognitiva del Homo sapiens y se pone el objetivo de identificar las condiciones biológicas universales de la variabilidad de los comportamientos estéticos. Los tres principales programas de investigación dedicados a la naturalización de lo estético son: la «estética empírica», la «neuroestética» $y$ la «estética evolutiva».

Heredera de la estética psicológica de Gustav Theodor Fechner, la estética «empírica» o «experimental» ha conocido una profunda renovación con los trabajos de Daniel Berlyne convirtiéndose en los últimos años en parte integrante del programa más general de las ciencias cognitivas (Pelowsky et al. 2016). Actualmente se encuentra en curso una fase de reformulación de los presupuestos teóricos del enfoque Fechner-Berlyne a partir de los elementos que la hipótesis estándar resulta incapaz de integrar, como la multiplicidad de 
los niveles cognitivos comprometidos en la evaluación de una obra de arte (Leder y Nadal 2014) y la variabilidad contextual e histórica de las preferencias estéticas (Bullot y Reber 2013).

La "neuroestética", término utilizado por primera vez por Semir Zeki para indicar el estudio de las relaciones neuronales de la experiencia visual de una obra de arte (Zeki 1999), en los últimos años ha experimentado un fuerte desarrollo gracias a la utilización de técnicas de visualización cerebral y a nuevas perspectivas teóricas más sensibles a la compleja naturaleza multimodal de la cognición estética. Nuevas investigaciones experimentales han extendido los factores causales definiendo la experiencia estética como un estado emergente que surge de la interacción de una triada de sistemas neuronales: los sistemas motores y somatosensoriales (Gales 2017), los circuitos de evaluación afectiva (Brown, et al. 2011) y aquellos otros responsables de la categorización conceptual. En esta dirección, la neuroestética se ha convertido progresivamente en un ámbito de investigación heterogéneo en el que confluyen varias hipótesis de trabajo (Chatterjee y Vartanian 2014; Huston et al. 2018).

Como se ha mencionado anteriormente, la «estética evolutiva» es un programa de investigación neodarwinista que, en el marco conceptual de la Síntesis Moderna, se pone como objetivo reconducir la complejidad de los fenómenos estéticos a caracteres biológicos, comportamentales y psicológicos innatos, seleccionados a lo largo de la historia evolutiva humana por su valor directamente adaptativo (Rusch y Voland 2013). De ello resulta un enfoque filogenético con respecto a las preferencias estéticas humanas basado en algunas asunciones reduccionistas entre las cuales un modelo de percepción centrado en las características objetivas del estímulo y la correlación directa entre módulos cognitivos adaptativos y preferencias estéticas.

\section{LA VERSIÓN DE LA MENTE}

Además de un paradigma epistémico naturalista, perspectivas como aquellas de la neuroestética o de la estética experimental asumen implícitamente una tesis de tipo mentalista según la cual la apreciación de una obra de arte está determinada exclusivamente por los mecanismos internos de la arquitectura cognitiva humana. Esto se debe a que el programa de naturalización de la mente, que constituye el marco teórico general de las estéticas naturalistas, se basa en el enfoque de las ciencias cognitivas clásicas en el que la actividad mental consiste en una serie de procesos de elaboración interna de estímulos que mantienen una relación causal (input-output) con los acontecimientos externos. Según esta "nueva filosofía mental" (Descombes 1995: 26-33), la "vita cognitiva" se puede observar indirectamente a través de sus efectos externos o con un acceso directo por medio de la visualización cerebral.

Casi todas las perspectivas naturalistas en estética adoptan esta «perspectiva internalista» sobre el funcionamiento de la cognición humana, tanto en una versión «representacionalista», según la cual la experiencia estética tiene su origen y realización en la activación de algún proceso neuronal o módulo cognitivo, como en una «somatosensorial», según la cual la apreciación estética es un producto de las modificaciones inducidas en el cuerpo por el estado emocional. La versión más radical del internalismo - suscrita, por ejemplo, por el enfoque estándar en neuroestética y por la estética psicoevolutiva- es la que se encuentra basada en la tesis de que no sólo los pensamientos, las emociones y las sensaciones son implementados en el sistema nervioso, sino que este último constituye la condición suficiente para la realización de una mente (Koch 2004: 9). La experiencia estética tiene su origen y realización en la activación de algún proceso neuronal y su duración fisiológica corres- 
ponde a los pocos cientos de milisegundos necesarios para realizar las conexiones neuronales. En lugar de los mecanismos superiores de cálculo e inferencia por obra de las capas más superficiales de la corteza cerebral, la versión somatosensorial se concentra, en cambio, en los niveles más bajos y antiguos de nuestro cerebro como la amígdala y el hipotálamo, responsables de los sistemas de regulación biológica corporal y de las respuestas afectivas. La vida mental se describe desde abajo como una producción de imágenes mentales referidas al sí-mismo, producto consciente de las modificaciones inducidas en el cuerpo por el estado emocional.

En la composición de un modelo estético, una versión internalista de la mente es compatible con una concepción de lo estético tanto cognitivista como emotivista. Ambas concepciones comparten, en efecto, un cuadro dualista que ve la oposición entre un componente interno y otro externo de los comportamientos humanos, una vida mental y social de los individuos, un componente físico y otro psíquico de la experiencia. En particular, la versión internalista de la mente se muestra solidaria con la doctrina modernista en el origen de la despotenciación de la dimensión antropológica de lo estético observado anteriormente. La noción de percepción estética basada en el modelo estímulo-respuesta de la psicología experimental de la tradición fechneriana es un caso paradigmático de esta solidaridad. Al reducir el componente social e institucional de los comportamientos estético-expresivos a procesos causales que se realizan en la oscuridad de nuestra caja craneal, la adopción de una versión internalista de la mente es responsable, sobre todo en las perspectivas naturalistas, de una insularización de lo estético ${ }^{11}$ y de una subde-

\footnotetext{
${ }^{11}$ Como ya observaba en 1934 John Dewey, existe una conexión estrecha entre una versión internalista de la mente y la insularización de lo estético: "This conception of mind as an isolated being underlies the conception that esthetic experience is
}

terminación de los procesos culturales reducidos a elementos lógicamente secundarios respecto a las operaciones cognitivas básicas de una mente individual, ahistórica y carente de contexto ecológico. De ahí el reciente surgimiento de estudios que vuelven a pensar la complejidad contextual de lo estético tomando en consideración elementos como la vivencia emocional subjetiva, la variabilidad biográfica o la historicidad de la visión dentro de un paradigma amplio y situado de la cognición humana como una adaptación socialmente distribuida que depende de la situación en la que se encuentra y actúa (Robbins y Aydede (2009); para un enfoque "situado" con respecto a la estética: Manzotti 2011).

\section{UN MAPA CONCEPTUAL}

Sobre la base del análisis realizado hasta ahora, es posible construir un mapa conceptual de los diferentes modelos de lo estético adoptados por las ciencias humanas contemporáneas. En sus investigaciones cualitativas o experimentales, los diferentes enfoques se basan, en su casi totalidad, en modelos estéticos que se componen de elementos teóricos dispuestos sobre ejes contrarios. En particular, hemos podido observar cómo los principales modelos estéticos surgen de la combinación de elementos compositivos que se establecen alrededor de dicotomías profundamente arraigadas en el pensamiento filosófico occidental. 


\begin{tabular}{|c|c|}
\hline Componentes de un modelo estético & Pares de dicotomías \\
\hline \multirow{2}{*}{ Concepción de lo estético } & Emotivismo \\
\cline { 2 - 2 } & Cognitivismo \\
\hline \multirow{2}{*}{ Paradigma epistémico } & Naturalización \\
\cline { 2 - 2 } & Culturalismo \\
\hline \multirow{2}{*}{ Versión internalista de la mente } & Representacionalista \\
\cline { 2 - 2 } & Somatosensorial \\
\hline
\end{tabular}

Al proyectar esta tabla sobre los ejes de un modelo cartesiano de tres dimensiones, obtenemos una visualización espacial del debate contemporáneo sobre la estética en las ciencias humanas. En el sistema de oposición representado en la figura, cada modelo estético es el resultado de la combinación de una concepción de lo estético, de un paradigma epistémico y de una versión de la mente.

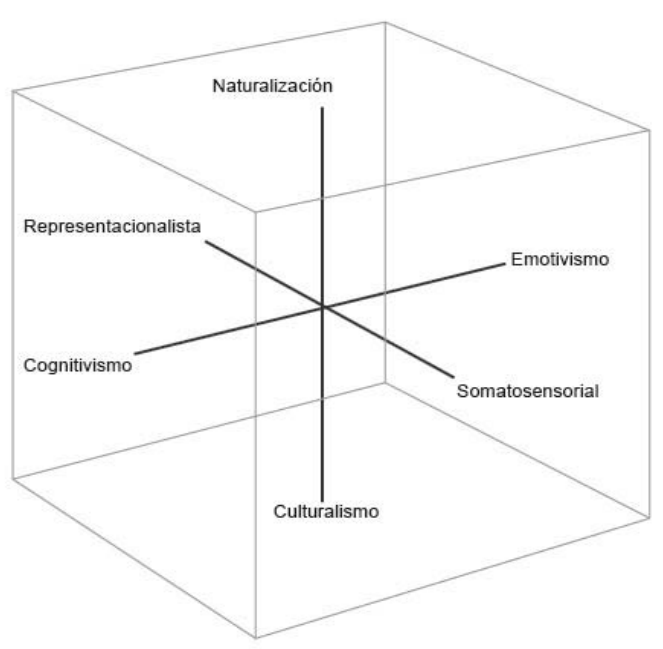

Ahora se tratará de colocar en esta topología conceptual de tres dimensiones los diferentes modelos estéticos. El paradigma dominante de la estética evolutiva se sitúa, por ejemplo, en el espacio que surge de la combinación de una concepción cognitivista de lo estético, una episteme naturalista y una versión de la mente representacionalista. En el mismo cuadrante podemos situar la neuroestética de Semir Zeki que, mediante una estrategia naturalizante radical, define lo estético como una forma de tratamiento de la información visual. La obra de arte se reconduce a componentes perceptuales estructurales como color, forma, movimiento, colocados en relación directa con una específica área especializada de la corteza visual primaria. Al permanecer dentro de un paradigma epistémico naturalista, la reciente teoría neurocientífica de David Freedberg y Vittorio Gallese parece integrarse perfectamente con una concepción emotivista. Poniendo el acento en el poder empático de las imágenes y en el nivel subsimbólico, ambos autores adoptan una versión somatosensorial de la mente con la intención de mostrar cómo en la percepción estética —en cuanto activación de "emociones empáticas imitativas"- está en juego una simulación automática de las acciones y de las sensaciones representadas a través de la activación de las mismas redes neuronales implicadas (Freedberg y Gallese, 2007).

Del lado culturalista de la falla epistémica, la teoría del objeto artístico de Gell (1998) se centra en la red de relaciones sociales en la que el artefacto se inserta y en los mecanismos de abducción a través de los cuales es posible deducir las intencionalidades delegadas que convierten el objeto en un agente social en un nivel de paridad con respecto a los individuos. La propuesta culturalista de Gell se basa pues en un modelo que asocia una concepción cognitivista de lo estético a una versión representacionalista de la mente derivada de la asunción de un innata «psicología folk» que guía los procesos 
cognoscitivos (derivada de la antropología cognitiva de Boyer 1996). También la estética filosófica no está exenta de la adopción implícita de modelos estéticos, ni es un ejemplo la estética analítica estadounidense que, al menos allí donde se ha concebido tradicionalmente como un "análisis de los conceptos y principios de la crítica” (Isenberg 1987: 128), se puede colocar en nuestro mapa en la intersección de un paradigma culturalista, una versión representacionalista de la mente y una concepción cognitivista de lo estético.

Este mapa conceptual no es más que un intento de poner orden entre las diferentes perspectivas sobre lo estético que han surgido en los últimos años en el seno de las diversas ciencias humanas. Destacando los puntos de contacto y los de conflicto, sacando a la luz afinidades teóricas inesperadas, haciendo emerger algunos supuestos teóricos inexplorados, el mapa tiene la única función de proporcionar un instrumento operativo y analítico. Poblar el mapa de modelos estéticos, encontrar para cada uno de ellos un lugar conceptual, no es más que un primer paso, humilde y conceptualmente parsimonioso, para un programa mucho más ambicioso, la formulación de una antropología general de lo estético.

\section{REFERENCIAS}

ANDERSON, Richard L. (1990), Calliope's Sisters: A Comparative Study of Philosophies of Art (Englewood Cliffs, N.J.: Prentice-Hall).

AYER, Alfred Jules (1936), Language, truth and logic (London: Victor Gollancz). BARKow, Jerome H., Cosmides, Leda \& ToOby, John (eds.) (1992), The Adapted Mind: Evolutionary Psychology and the Generation of Culture (New York: Oxford University Press).

BARTALeSI, Lorenzo (2012), Estetica evoluzionistica. Darwin e l'origine del senso estetico (Roma: Carocci).
(2013), "Quale storia naturale per l'estetico? L'ipotesi darwiniana rivisitata", Rivista di estetica, 54, 3: 7-27.

__ (2017), Antropologia dell'estetico (Milano: Mimesis).

BARTHES, Roland (1964a), Essais critiques (Paris: Éditions du Seuil)

(1964b), "L'activité structuraliste", en Barthes (1964a: 211-219).

BeLl, Clive (1914), Art (New York: Stokes).

BOAS, Franz ([1927] 1951), Primitive art (Irvington-on-Hudson, N.Y.: Capitol Publishing).

BOURDIEU, Pierre (1979), La distinction. Critique sociale du jugement (Paris: Éditions de Minuit).

BOYER, Pascal (1996), "What makes anthropomorphism natural: intuitive ontology and cultural representations", Journal of the Royal Anthropological Institute, 2: 83-97.

Brown, Steven, GaO, Xiaoqing, Tisdelle, Loren, Eickhoff, Simon B., LIOTTI, Mario (2011), "Naturalizing aesthetics: Brain areas for aesthetic appraisal across sensory modalities", NeuroImage, 58: 250258.

BULlot Nicolas J. \& REBER, Rolf (2013), “A psycho-historical research program for the integrative science of art", Behavioral and Brain Sciences, 36 (2): 163-180.

CARLSON, Allen (1999), Aesthetics and the environment. The appreciation of nature, art and architecture (London-New York: Routledge).

Chatterjee, Anjan \& Vartanian, Oshin (2014), "Neuroaesthetics", Trends in Cognitive Sciences, 18, 7: 370-375.

Conkey, Margaret W. \& HASTORF, Christine Ann (eds.) (1990), The Uses of Style in Archaeology, (Cambridge: Cambridge University Press).

Coote, Jeremy \& Shelton, Anthony (eds.) (1992), Anthropology, Art, and Aesthetics (Oxford: Clarendon Press).

CURRIE, Greg (2016), "Aesthetic Explanation and the Archaeology of Symbols”, British Journal of Aesthetics, 56, 3: 233-246. 
D’Errico, Francesco \& Henshilwood, Christopher S. (2011), Homo Symbolicus: The Dawn of Language, Imagination and Spirituality (Amsterdam-Philadelphia: John Benjamins Publishing).

DESCOMBES, Vincent (1995), La denrée mentale (Paris: Éditions de Minuit). DEWEY, John ([1934] 1980), Art as experience (New York: Perigee Books).

Di LiBerti, Giuseppe (2017), "Le fait esthétique", Archives de Pilosophie, 80, 2: 295-310.

DisSANAYAKE, Ellen (2000), Art and intimacy: How the arts began (Seattle: University of Washington Press).

_ (2013), "Genesis and development of 'Making Special': Is the concept relevant to aesthetic philosophy?", Rivista di Estetica, 54, 3: 83-98.

ElKINS, James \& MonTgomery, Harper (eds.) (2013), Beyond the Aesthetic and the Anti-Aesthetic (Philadelphia: Pennsylvania State University).

FREEDBERG, David \& GALLESE, Vittorio (2007), "Motion, emotion and empathy in esthetic experience", Trends in Cognitive Sciences, 11, 5: 197-203.

GALLESE, Vittorio (2017), "Visions of the body: Embodied simulation and aesthetic experience", Aisthesis, 10 (1): 41-50.

GARRONI, Emilio (1992), Estetica. Uno sguardo-attraverso (Milano: Garzanti).

GELL, Alfred (1992), “The technology of enchantment and the enchantment of technology", en Coote \& Shelton (1992: 40-66).

(1998), Art and agency (Oxford: Clarendon Press).

Goodman, Nelson (1968), Languages of Art: An Approach to a Theory of Symbols (Indianapolis: The Bobbs-Merrill Company).

HaHn, Lewis E. (ed.) (1992), The philosophy of A. J. Ayer (La Salle IL: Open Court).

Hobart, Angela \& KAPFerer, Bruce (eds.) (2005), Aesthetics in Performance: Formations of Symbolic Construction and Experience (New York: Berghahn Books).

Huston, Joseph P., Nadal, Marcos, Mora, Francisco, Agnati, Luigi F., Cela-Conde, Camilo J. (eds.) (2018), Art, Aesthetics, and the Brain (Oxford: Oxford University Press).

InGOLD, Tim (ed.) (1996), Key Debates in Anthropology, (London: Routledge).
ISENBERG, Arnold (1987), "Analytical Philosophy and the Study of Art", The Journal of Aesthetics and Art Criticism, 46: 125-136.

JoPlING, Carol F. (ed.) (1971), Art and aesthetics in primitive societies, (New York: E. P. Dutton)

Kieran, Matthew \& MCIver Lopes, Dominic (2006), Knowing Art. Essays in Aesthetics and Epistemology (Dordrecht: Springer).

KIVY, Peter (1992), “"Oh boy! You too!»: Aesthetic emotivism reexamined”, en Hahn (1992: 309-325).

KocH, Christof (2004), The quest for consciousness: A neurobiological approach (Englewood, CO: Roberts \& Company).

LAWAL, Babatunde (1974), "Some Aspects of Yoruba Aesthetics", British Journal of Aesthetics, XIV, 3: 239-249.

LEDER, Helmut \& NADAL, Marcos (2014), “Ten years of a model of aesthetic appreciation and aesthetic judgments: The aesthetic episode. Developments and challenges in empirical aesthetics", British Journal of Psychology, 105: 443-464.

LeVI-STrauss, Claude (1950), "Introduction à l'œuvre de Marcel Mauss", en Mauss (1950: IX-LII).

(1958a), Anthropologie structurale (Paris: Plon).

_ (1958b), "La notion de structure en ethnologie", en Lévi-Strauss (1958a: 303-352).

LORBLANCHET, Michel \& BAHN, Paul G. (2017), The first artists. In search of the world's oldest art (London: Thames \& Hudson).

MANZOTTI, Riccardo (ed.) (2011), Situated aesthetics: Art beyond the skin (Charlottesville, VA: Imprint Academic).

MAQUET, Jacques J.P. (1986), The Aesthetic Experience. An Anthropologist Looks at the Visual Arts (New Haven-London: Yale University Press).

MAUSS, Marcel ([1926] 1967), Manuel d'ethnographie (Paris: Éditions Payot). (1950), Sociologie et anthropologie (Paris: PUF).

MORPHY, Howard (1996), "Aesthetics is a cross-cultural category. For the motion”, en Ingold (1996: 255-260). 
PANEBIANCO, Fabrizio \& SERRELli, Emanuele (eds.) (2016), Understanding Cultural Traits (Chaim: Springer).

Pelowsky, Matthew, Markey, Patrick S., Lauring Jon O. \& Leder Helmut (2016), "Visualizing the Impact of Art: An Update and Comparison of Current Psychological Models of Art Experience", Frontiers in Human Neuroscience, 4/26: 1-21.

PORTERA, Mariagrazia \& BARTALESI, Lorenzo (2016), Aesthetic preferences: An evolutionary approach, en Panebianco \& Serrelli (2016: 375-388).

RoBbINS, Philip \& AydEDE, Murat (eds.) (2009), The Cambridge Handbook of Situated Cognition (Cambridge: Cambridge University Press).

Robson, Jon (2012), “Aesthetic Testimony”, Philosophy Compass, 7, 1: 1-10. RUSCH, Hannes \& VOLAND, Eckart (2013), "Evolutionary Aesthetics: an Introduction to Key Concepts and Current Issue", Aisthesis, 6, 2: 113-133.

SCHAEFFER Jean-Marie (1992), L'art de l'âge moderne. L'esthétique et la philosophie de l'art du XVIIIe siècle à nos jours (Paris: Gallimard).

_ (2015a), Lettre à Roland Barthes (Paris: Editions Thierry Marchaisse). (2015b), L'expérience esthétique, Gallimard, Paris.

STOUT, David B. (1971), "Aesthetics in primitive society", en Jopling (1971: 30-34).

TALON-HugOn, Carole (2014), L'art victime de l'esthétique (Paris: Hermann). THompson, Robert Farris (1971), Aesthetics in Traditional Africa, en Jopling (1971: 374-381).

Vogel, Susan M. (1980), Beauty in the eyes of the Baule. Aesthetics and Cultural Values (Philadelphia: Institute for the Study of Human Issues).

VOLAND, Eckart, GrAMmER, Karl (eds.) (2003), Evolutionary Aesthetics (Heidelberg: Springer).

WeLSCH, Wolfgang (2013), "L’origine animale dell'estetica", Rivista di estetica, 54, 3: 181-206.

White, Randall (1992), "Beyond Art: Toward an Understanding of the Origins of Material Representation in Europe", Annual Review of Anthropology, 21: 537-564.

ZEKI, Semir (1999), Inner vision: an exploration of art and the brain (Oxford: Oxford University Press). 